\title{
Failing the frail: The need to broaden the COVID-19 case definition for geriatric patients
}

\author{
Authors: Clare Hunt, ${ }^{A *}$ Flora Olcott, ${ }^{B *}$ George Williams ${ }^{C}$ and Terrence Chan ${ }^{D}$
}

The older population has a high mortality with COVID-19 and this cohort often presents atypically with infection. This study compares presenting complaints and observations of older patients with COVID-19 against the established case definition to determine whether the case definition should be broadened to better identify SARS-CoV-2 infection in this age group.

This retrospective observational study analysed the presenting complaints and observations of people aged 70 years and over who were admitted to a district general hospital with confirmed SARS-CoV-2 infection from March to May 2020.

Out of 225 patients, only $11.5 \%$ presented with the trio of cough, fever and breathlessness; $30.2 \%$ did not present with any of these symptoms $(p<0.001)$. The most frequent atypical complaints were delirium (25\%), general malaise (20\%) and falls (19\%). Only $32.4 \%$ recorded a temperature $\geq 37.6^{\circ} \mathrm{C}$ on admission while $20.4 \%$ were hypothermic with a temperature $<36.4^{\circ} \mathrm{C}(\mathrm{p}=0.0003)$.

A significant proportion of older patients with COVID-19 presented with non-specific symptoms and observations. The high proportion of falls and delirium emphasises the need for early geriatrician input, awareness of COVID-19 as a differential for confusion in older patients and to include falls in the case definition for COVID-19 in the older population.

KEYWORDS: COVID-19, geriatric medicine, presenting complaints, falls, delirium

DOI: 10.7861/clinmed.2021-0308

\section{Introduction}

The novel virus, SARS-CoV-2, and resultant COVID-19 global pandemic have highlighted the omnipresent threat of emerging

Authors: ${ }^{\mathrm{A}} \mathrm{ST} 7$ in geriatric medicine, Maidstone and Tunbridge Wells NHS Trust, Tunbridge Wells, UK; ${ }^{\text {B }}$ foundation year-2 doctor, Lewisham and Greenwich NHS Trust, London, UK; ${ }^{C_{\text {foundation }}}$ year-1 doctor, Barts Health NHS Trust, London, UK; D foundation year-2 doctor, Lewisham and Greenwich NHS Trust, London, UK; *joint first authors infectious diseases. SARS-CoV-2 has a high level of transmissibility and is associated with significant morbidity and mortality. The older population are particularly at risk, with the estimated agespecific infection fatality ratio increasing with age from $1.4 \%$ at 65 years to $15 \%$ at 85 years. ${ }^{1,2}$

Since 18 May 2020, the three main symptoms indicating a possible COVID-19 case, as outlined by Public Health England, are a new continuous cough, temperature $\geq 37.8^{\circ} \mathrm{C}$, or loss of or change in sense of smell or taste. The majority of public health campaigns, as well as criteria for community testing, focus on these three symptoms. However, it is acknowledged that older people often present with infections atypically and this is likely the case for COVID-19. ${ }^{3}$ This may have significant implications for case identification, as well as treatment and prevention of outbreaks within the hospital and community setting.

Given the particular danger that COVID-19 poses to this age group, it is essential that we establish effective diagnosis, management and infection-control methods. We, therefore, aim to characterise the clinical features of older people with COVID-19 admitted to a district general hospital during the initial stages of the pandemic to improve recognition of COVID-19 in this population.

\section{Methods}

\section{Study design}

We performed a retrospective observational study of patients aged 70 years and over who were admitted with COVID-19 to a district general hospital in Kent, south-east England, between 15 March 2020 and 31 May 2020. Only those that tested positive for SARS-CoV-2 RNA via reverse transcriptase-polymerase chain reaction (RT-PCR) were included. Those that tested positive for SARS-CoV-2 RNA 7 days or more into their hospital admission were excluded due to the possibility of hospital-acquired infection. For those patients readmitted during the study period following an earlier diagnosis of COVID-19 infection, only data from their initial hospital admission were included.

Clinical characteristics and observations on presentation to hospital were obtained from the initial hospital electronic record. Presenting complaints documented by the emergency department (ED) triage were analysed and symptoms assigned to one or more of 14 categories (Fig 1). Presenting complaints of confusion and reduced consciousness were combined under 


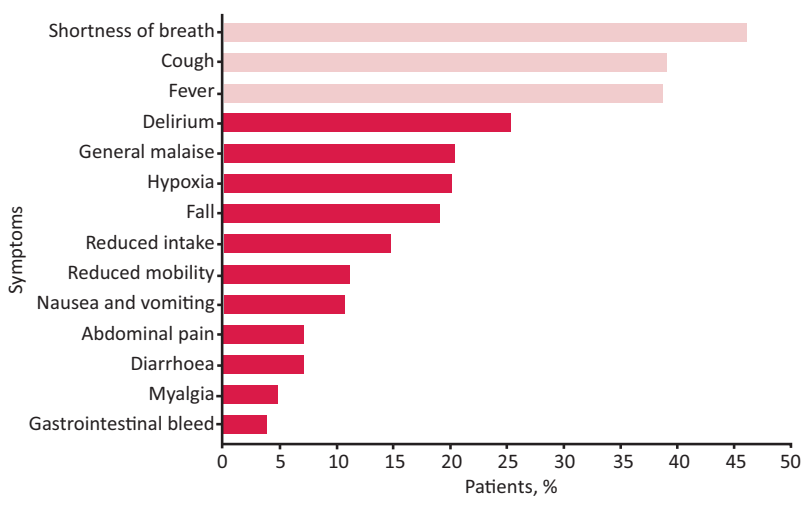

Fig 1. Symptom categories and occurrence.

a diagnosis of delirium and categorised accordingly. Clinical observations of temperature, oxygen saturation and respiratory rate were collected and categorised. Oxygen requirement on admission was also noted.

Outcomes were recorded as either death during admission or discharge.

\section{Statistical analysis}

Median and interquartile range (IQR) were used to report nonparametric continuous data, and mean and standard deviation (SD) were used to report parametric continuous data. Categorical data were described using percentages. Fisher's exact test and Mann-Whitney $U$ test were used to compare categorical data and continuous data, respectively.

Univariate analysis of outcomes between groups was performed using logistic regression and reported as odds ratio (OR) with $95 \%$ confidence interval (CI). We also selected variables (shortness of breath, respiratory rate $>20$ breaths per minute, oxygen saturations $<92 \%$, cough, hypoxia, falls, delirium and accommodation type) to be included in a multivariate analysis, adjusted for age and sex. These variables were selected based on previous studies that have shown to predict mortality. ${ }^{4-6}$

Data manipulation and analysis were performed using R v4.0.0 (RStudio, Boston, USA).

\section{Results}

A total of 225 patients were included: 120 (53.3\%) patients were men and $105(46.7 \%)$ were women, with a mean age of 83 years (SD 82-84). One-hundred and fifty-four (68.4\%) patients were from their own home, 49 (31.8\%) from a residential home and 22 $(9.8 \%)$ from a nursing home (Table 1$)$.

On admission, patients within this cohort often presented atypically. Only $26(11.5 \%)$ patients presented with the trio of cough, fever and breathlessness; 68 (30.2\%) did not present with any of these symptoms when compared with the rest of the cohort $(p<0.001)$. The most frequent atypical complaints were delirium $(n=57 ; 25.3 \%)$, general malaise $(n=46 ; 20.4 \%)$ and falls $(n=43$; $19.1 \%$; Fig 1). Of those who presented with a fall, 34 (79.1\%) patients were from their own home while four $(1.8 \%)$ were from sheltered accommodation. Twelve (27.9\%) patients who presented with a fall also had delirium.
Table 1. Demographics and clinical characteristics of patients on admission

$\begin{array}{ll}\text { Age, years, mean (standard deviation) } & 83(82-84) \\ \text { Sex, } \mathrm{n}(\%) & \\ \quad \text { Male } & 120(53.3) \\ \text { Female } & 105(46.7) \\ \text { Accommodation, } \mathrm{n}(\%) & \\ \quad \text { Own home } & 154(68.4) \\ \text { Sheltered accommodation } & 11(4.9) \\ \text { Residential home } & 49(31.8) \\ \quad \text { Nursing home } & 22(9.8) \\ \text { Clinical presentation, } \mathrm{n}(\%) & \\ \quad \text { Fever }>37.6^{\circ} \mathrm{C} & 87(38.8) \\ \quad \text { Respiratory rate }>20 \text { breaths per minute } & 95(42.4) \\ \text { Oxygen saturation }<92 \% & 51(22.8)\end{array}$

Normothermia or hypothermia and an oxygen saturation of $>92 \%$ were frequently observed in these patients. Only $73(32.4 \%)$ patients recorded a temperature $\geq 37.6^{\circ} \mathrm{C}$ on presentation while $127(56.4 \%)$ presented with normothermia or hypothermia $(p<0.0001)$. Forty-six $(20.4 \%)$ patients were hypothermic with a temperature $<36.4^{\circ} \mathrm{C}$ and $81(36.0 \%)$ recorded a normal temperature of $36.5-37.5^{\circ} \mathrm{C}$. Oxygen saturation data was available for 198 patients, with 147 (74.2\%) patients having an oxygen saturation of $>92 \%$ compared with $52(26.2 \%)$ who had an oxygen saturation of $<92 \%$ on admission ( $<<0.0001$; Table 2).

The in-hospital mortality within this cohort was high; 105 $(46.7 \%)$ patients died by the end of the study period. Patients from nursing or residential homes were also more likely to have an adverse outcome (OR 2.48; 95\% CI 1.40-4.46; $p=0.002$ ). While not statistically significant, male patients were less likely to survive than female patients (OR 1.43; 95\% CI 0.84-2.44; $\mathrm{p}>0.05$ ). Shortness of breath, low oxygen saturation and high respiratory rate predict in-hospital mortality in univariate analysis when adjusted for age and sex. Other documented presenting complaints of patients did not predict in-hospital mortality rates within this cohort. Respiratory rate $>20$ breaths per minute and admission from nursing or residential homes were more likely to have an adverse outcome on multivariate analysis (Table 2).

\section{Discussion}

Our study shows that the majority of older adults did not present with the trio of fever, cough and shortness of breath. While they may suffer one of these symptoms, a greater proportion of this cohort present with additional atypical or non-specific symptoms such as falls, confusion, drowsiness or general malaise. This stresses the need to consider COVID-19 as part of the differential diagnosis in these presenting complaints and the importance of a thorough history-taking to assess for possible underlying causes.

In particular, 19\% presented following a fall. Previous observational studies have noted falls as a common atypical presentation for infections in older adults. ${ }^{7}$ Falls in the context of SARS-CoV-2 infection may occur for a variety of reasons including confusion, myalgia or hypoxia. One consequence of falls is hip fracture and, not surprisingly, patients with concurrent SARS-CoV-2 infection have a lower 30-day survival rate than those without. $^{3}$ 
Table 2. Outcomes between survivors and non-survivors

\begin{tabular}{|c|c|c|c|c|}
\hline & Univariate analysis & & Multivariate analysis & \\
\hline & OR $(95 \% \mathrm{CI})$ & p value & OR $(95 \% \mathrm{CI})$ & $\mathrm{p}$ value \\
\hline Male & $1.43(0.85-2.44)$ & 0.18 & $1.66(0.86-3.25)$ & 0.13 \\
\hline Age & $1.04(0.99-1.08)$ & 0.075 & $1.05(0.99-1.11)$ & 0.079 \\
\hline Admission from nursing or residential home & $2.48(1.40-4.47)$ & 0.002 & $2.15(1.05-4.47)$ & 0.038 \\
\hline Fever, temperature $>37.6^{\circ} \mathrm{C}$ & $1.01(0.59-1.74)$ & 0.95 & & \\
\hline Respiratory rate $>20$ breaths per minute & $4.11(2.28-7.55)$ & $<0.0001$ & $4.16(2.08-8.59)$ & $<0.0001$ \\
\hline Oxygen saturation $<92 \%$ & $2.16(1.11-4.29)$ & 0.022 & $1.20(0.53-2.71)$ & 0.67 \\
\hline Shortness of breath & $1.84(1.08-3.15)$ & 0.023 & $1.15(0.55-2.37)$ & 0.71 \\
\hline Cough & $0.58(0.34-1.01)$ & 0.053 & $0.61(0.30-1.22)$ & 0.17 \\
\hline Delirium & $1.25(0.69-2.29)$ & 0.46 & $1.43(0.66-3.10)$ & 0.36 \\
\hline General malaise & $1.06(0.55-2.03)$ & 0.86 & & \\
\hline Fall & $0.54(0.26-1.07)$ & 0.088 & $0.64(0.27-1.49)$ & 0.31 \\
\hline Reduced intake & $0.71(0.33-1.49)$ & 0.37 & & \\
\hline Reduced mobility & $0.89(0.38-2.04)$ & 0.78 & & \\
\hline Nausea and vomiting & $0.43(0.16-1.04)$ & 0.078 & & \\
\hline Abdominal pain & $0.89(0.24-1.05)$ & 0.77 & & \\
\hline Diarrhoea & $0.67(0.22-1.86)$ & 0.44 & & \\
\hline Myalgia & $0.95(0.26-3.24)$ & 0.93 & & \\
\hline Gastrointestinal bleed & $0.32(0.14-1.34)$ & 0.16 & & \\
\hline
\end{tabular}

This finding is supported by another recent study that has shown falls to be among the most prevalent atypical symptoms of SARS$\mathrm{CoV}-2$ infection in residents of nursing homes in France. ${ }^{8}$ Given that nearly one in five patients in our cohort presented with falls, this highlights the importance of including falls as a feature of SARS-CoV-2 infection in older adults.

Delirium, characterised by disturbed consciousness and new cognitive dysfunction, has also proven a prominent feature of SARS-CoV-2 infection in older adults in both hospital and community settings, particularly in the context of frailty. ${ }^{9-11}$ Our study demonstrates that delirium is the most common atypical presentation in this cohort, a finding confirmed across other larger studies. ${ }^{11}$ The presence of delirium has been associated with poorer outcomes than the more typical, core symptoms. ${ }^{11}$ As of October 2020, this has been reflected in the UK government guidance and has since been incorporated into official guidelines, such as that of SIGN. ${ }^{13}$ These state that the presence of delirium in a patient presenting to hospital should prompt clinicians to suspect and test for SARS-CoV-2, even if it is an isolated symptom.

It is, therefore, vital that history-taking is accurate and collateral histories are obtained to provide a clear picture of events preceding and precipitating the fall or confusion. We believe this emphasises the need for early geriatrician assessment to help support better care for older adults with SARS-CoV-2 infection.

Older patients also have differing clinical observations on presentation to hospital than younger patients. A minority of this cohort had a fever on admission, with a significant proportion recording hypothermia; these findings are consistent with previous COVID-19 studies that identified fever to be less common with advanced age. ${ }^{11}$ This creates difficulty in the triage of patients and is likely to have had implications on infection control within the hospital. We believe that accurate rapid diagnostic testing of older patients in the ED will be beneficial in maximising infection control and ensuring patient safety.

It is important to consider the societal constraints created by the pandemic and the subsequent exacerbation of frailty in this cohort. Imposed national lockdowns and 'shielding' led to reduced access to social services, and occupational and physical therapies as well as reduced rates of physical exercise and social interaction, all of which contribute to an increased falls risk and reduced physiological reserve in this population.

Frailty is not just limited to the older adult population, it is important to consider the possibility of atypical presenting complaints in frail younger adults. Studies on COVID-19 in this cohort are more limited when compared with older adults and given the association between frailty and adverse outcome, further research into COVID-19 in the younger frail adult population is required.

\section{Limitations}

Our study was limited by the retrospective use of hospital electronic records to ascertain the initial patient observations and presenting complaint. It did not account for whether patients may have received paracetamol prior to admission, potentially resulting in normothermia or hypothermia. Given the high prevalence of cognitive impairment or delirium in this age group, many patients would not have been able to reliably report their symptoms and collateral histories may not have been acquired in triage. 
The relatively small sample size of this study is also a limitation. A number of older patients in whom SARS-CoV-2 infection was clinically suspected were not included due to a negative RT-PCR test result. One potential explanation could be inadequate swabbing technique during the initial stage of the first wave.

\section{Conclusion}

Our study shows that a significant proportion of older people with COVID-19 presented to hospital with non-specific or atypical symptoms and observations. The relatively high number of falls and delirium precipitating hospital attendance in this cohort stresses the need to consider a diagnosis of COVID-19 while the disease remains prevalent. This also emphasises the need for early geriatrician input and to include falls in the case definition for COVID-19 in the older population.

\section{Summary}

\section{What is known?}

The older population have been shown to have a high mortality with COVID-19.

This cohort often present atypically with infections and there is, therefore, a need to increase understanding of how COVID-19 affects this population.

\section{What is the question?}

To assess whether older people admitted to hospital present with symptoms different to those listed in the COVID-19 case definition.

\section{What was found?}

Few patients over 70 years with COVID-19 presented with the trio of cough, breathlessness and fever, and over $30 \%$ presented with none of these.

Only $32.4 \%$ of older patients present with fever, making temperature an unreliable indicator of COVID-19 infection in this cohort.

A fifth (19\%) of patients in our cohort presented to hospital following a fall.

\section{What is the implication for practice now?}

Clinical suspicion for COVID-19 infection is needed in patients presenting with falls and confusion, even if clinical observations are normal.

Obtaining a clear history of any fall and preceding events is vital. These findings emphasise the importance of early geriatrician involvement in the care of older patients in hospital. The need to acknowledge falls in the case definition for SARSCoV-2 infection.

\section{References}

1 Docherty AB, Harrison EM, Green CA et al. Features of 20133 UK patients in hospital with COVID-19 using the ISARIC WHO Clinical Characterisation Protocol: prospective observational cohort study. BMJ 2020;369:m1985.

2 Levin AT, Hanage WP, Owusu-Boaitey N et al. Assessing the age specificity of infection fatality rates for COVID-19: systematic review, meta-analysis, and public policy implications. Eur ] Epidemiol 2020;35:1123-38.

3 Wester AL, Dunlop O, Melby KK, Dahle UR, Wyller TB. Age-related differences in symptoms, diagnosis and prognosis of bacteremia. BMC Infectious Diseases 2013;13:346.

4 Zheng Z, Peng F, Xu B et al. Risk factors of critical \& mortal COVID-19 cases: A systematic literature review and meta-analysis. J Infect 2020;81:e16-25.

5 Poloni TE, Carlos AF, Cairati M et al. Prevalence and prognostic value of Delirium as the initial presentation of COVID-19 in the elderly with dementia: An Italian retrospective study. EClinicalMedicine 2020:26:100490.

6 Shi L, Wang Y, Wang Y, Duan G, Yang H. Dyspnea rather than fever is a risk factor for predicting mortality in patients with COVID-19. J Infect 2020;81:647-79.

7 Hofman MR, van den Hanenberg F, Sierevelt IN, Tulner CR. Elderly patients with an atypical presentation of illness in the emergency department. Neth J Med 2017;75:241-6.

8 Blain $\mathrm{H}$, Gamon L, Tuaillon E et al. Atypical symptoms, SARS-CoV-2 test results and immunisation rates in 456 residents from eight nursing homes facing a COVID-19 outbreak. Age Ageing 2021;50:641-8.

9 National Institute for Health and Care Excellence. Delirium: prevention, diagnosis and management: Clinical guideline [CG103]. NICE, 2019.

10 Zazzara MB, Penfold RS, Roberts AL et al. Probable delirium is a presenting symptom of COVID-19 in frail, older adults: a cohort study of 322 hospitalised and 535 community-based older adults. Age Ageing 2021;50:40-8.

11 Millar JE, Neyton L, Seth S et al. Robust, reproducible clinical patterns in hospitalised patients with COVID-19. medRxiv 2020;2020.08.14. 20168088.

12 Shao S-C, Lai C-C, Chen Y-H et al. Prevalence, incidence and mortality of delirium in patients with COVID-19: a systematic review and meta-analysis. Age Ageing 2021;afab103. [Epub ahead of print].

13 Ellis G, Beattie ], Burns ] et al. COVID-19 position statement: Presentations and management of COVID-19 in older people in acute care. Scottish Government, 2021. www.sign.ac.uk/media/ 1826/presentations-and-management-of-covid-19-in-older-people-v2final.pdf

Address for correspondence: Dr Clare Hunt, Elderly Care Department, Tunbridge Wells Hospital, Tonbridge Road, Pembury, Tunbridge Wells, Kent TN2 4QJ, UK.

Email: clare.hunt1@nhs.net 